Service social

\title{
Parentalité et déficience intellectuelle : enjeux de l'intervention auprès des familles
}

\section{Élise Milot, Daniel Turcotte et Sylvie Tétreault}

Volume 60, numéro 1, 2014

Des enfants, des couples et des familles.

URI : https://id.erudit.org/iderudit/1025133ar

DOI : https://doi.org/10.7202/1025133ar

Aller au sommaire du numéro

Éditeur(s)

École de service social de l’Université Laval

ISSN

1708-1734 (numérique)

Découvrir la revue

Citer cet article

Milot, É., Turcotte, D. \& Tétreault, S. (2014). Parentalité et déficience intellectuelle : enjeux de l'intervention auprès des familles. Service social, 60(1), 49-71. https://doi.org/10.7202/1025133ar
Résumé de l'article

Dans les sociétés contemporaines, un nombre croissant de personnes vivant avec une déficience ou une lenteur intellectuelle choisissent de devenir parents. Cette recension des écrits, s'inscrivant dans le cadre d'une recherche doctorale, propose d'examiner différents aspects de cette parentalité particulière au Québec et ailleurs. Elle aborde également les défis et les enjeux de l'intervention éducative et psychosociale. Prenant appui sur ces éléments, elle propose des recommandations ou des stratégies pertinentes à mettre en place au Québec en vue de favoriser le bien-être de ces parents. 


\title{
Parentalité et déficience intellectuelle : enjeux de l'intervention auprès des familles
}

\author{
MILOT, Élise \\ Doctorante en service social \\ École de service social \\ Université Laval \\ TURCOTTE, Daniel \\ Professeur titulaire \\ École de service social \\ Université Laval \\ TÉTREAULT, Sylvie \\ Professeure titulaire \\ Faculté de médecine \\ Université Laval
}

\section{RESUME}

Dans les sociétés contemporaines, un nombre croissant de personnes vivant avec une déficience ou une lenteur intellectuelle choisissent de devenir parents. Cette recension des écrits, s'inscrivant dans le cadre d'une recherche doctorale, propose d'examiner différents aspects de cette parentalité particulière au Québec et ailleurs. Elle aborde également les défis et les enjeux de l'intervention éducative et psychosociale. Prenant appui sur ces éléments, elle propose des recommandations ou des stratégies pertinentes à mettre en place au Québec en vue de favoriser le bien-être de ces parents.

Mots-clés : parentalité, parents, déficience intellectuelle, soutien à la famille, protection de l'enfance, protection de la jeunesse, habiletés parentales, compétences parentales.

\begin{abstract}
In modern societies, an increasing number of adults living with an intellectual disability choose to become parents. This review of the literature, as an integral part of a doctoral research project, proposes to examine various aspects of this particular form of parenting in Quebec and elsewhere. It also addresses the challenges and issues of educational and psychosocial intervention. Based on these elements, recommendations or relevant strategies required to establish and promote the well-being of those parents in Quebec are being addressed.
\end{abstract}

Key words: parenting, intellectual disabilities, child welfare, parenting skills, child rearing, parenting abilities. 


\section{INTRODUCTION}

Dans la plupart des pays industrialisés, il y a eu une hausse croissante de la parentalité chez les personnes ayant une déficience intellectuelle (DI) au cours des trente dernières années (Llewellyn et al., 2010; National Council on Disability, 2012). À ce jour, aucune donnée épidémiologique ne permet de déterminer le nombre exact de parents ayant une DI au Québec ou au Canada. En Australie, leur prévalence est évaluée à $1 \%$ (McConnell et al., 2000). Or, cette estimation ne tient pas compte des parents dont les limitations cognitives et adaptatives sont caractéristiques d'une lenteur intellectuelle (LI), aux limites du diagnostic. Des experts sont d'avis que ces parents font partie d'une majorité invisible et inconnue des statistiques (Emerson et Brigham, 2013). D'ailleurs, de plus en plus d'études réfèrent à une population plus globale de parents ayant une DI ou une LI (Aunos et Pacheco, 2013 ; Azar et al., 2013). L'importance de bien identifier leurs besoins et d'adapter l'offre de services pour y répondre s'avère fondamentale.

Pour les personnes présentant des incapacités, le droit à l'exercice de la parentalité implique que des mesures appropriées soient mises en place pour les soutenir dans l'exercice de leurs responsabilités parentales (Organisation des Nations Unies [ONU], 2006). Dans le cas des parents présentant une DI, l'offre de services spécialisés vise à suppléer leurs limites pour leur offrir la possibilité d'élever leur enfant avec succès (Lightfoot et LaLiberte, 2011 ; MacLean et Aunos, 2010). Toutefois, la représentation de cette parentalité singulière pose des défis particuliers pour le développement de pratiques professionnelles appropriées (Coppin, 2001 ; Aunos et Feldman, 2002 ; Jöreskog et Starke, 2013).

Cet article propose d'examiner la réalité vécue par les parents ayant une DI en s'appuyant sur les écrits scientifiques et les rapports spécialisés sur le sujet. Pour guider la recherche documentaire, différentes bases de données ont été consultées soit : PsycINFO, Social Services Abstracts, Social Work Abstracts, Famili@, Cairn et Érudit. Différents mots-clés liés à la DI (intellectual disability, intellectual development disorder, mental retardation [ancienne appelation], learning disability et learning difficulty [appelation utilisée au Royaume-Uni]) ont été croisés avec la notion de parentalité (parenthood, parenting, parenting skills, parent child relationships et child rearing). En outre, la consultation de sites internet de centres de réadaptation en déficience intellectuelle et en troubles envahissants du développement (CRDITED) a permis l'accès à des rapports non publiés (littérature grise) sur l'expérience vécue par les parents et les intervenants québécois impliqués.

Les informations recueillies ont été ventilées sous quatre thèmes, lesquels font l'objet des différentes sections de cet article : (1) la réalité des enfants qui vivent dans une famille dont au moins un parent a une DI; (2) les différents facteurs caractérisant la réalité de ces parents; (3) les défis et enjeux de l'intervention éducative et psychosociale;(4) les stratégies visant à mieux soutenir ces parents. Le rationnel de ce choix se base sur le modèle de la parentalité des personnes présentant une DI de Feldman (2002), lequel s'inspire de modèles explicatifs reconnus (écologique, transactionnel et interactionnel). 


\section{La réalité des enfants dont les parents ont une DI}

Les études portant sur le développement des enfants qui vivent avec des parents présentant une DI rapportent des résultats dont les variations sont substantielles (Mildon et al., 2003). Certaines indiquent que ces enfants sont davantage à risque d'avoir un retard de développement qui se manifesterait plus particulièrement sur le plan langagier (Feldman et al., 1985 ; Pixa-Kettner, 1999) et qui aurait tendance à s'accentuer au fil des ans (Whitman et Accardo, 1990). Cette situation pourrait justifier la plus forte prévalence chez ces enfants d'un retard intellectuel ou de troubles d'apprentissage (Feldman et Walton-Allen, 1997 ; Booth et Booth, 1998). Or, une recension des recherches sur le sujet met en lumière qu'entre $46 \%$ et $91 \%$ des enfants de tels parents ont une intelligence moyenne et même supérieure (Aunos et al., 2003). Lorsque c'est le cas, ces enfants auraient davantage tendance à assumer le rôle de parent au sein de la famille ou à se rebeller contre l'autorité parentale (O'Neill, 1985 ; O'Neill, 2011 ; Feldman et Walton-Allen, 1997).

Les problèmes de comportement ou d'adaptation psychosociale seraient également plus présents chez ces enfants que dans la moyenne de la population (Seagull et Scheurer, 1986 ; Tymchuk et Feldman, 1991 ; Feldman et Walton-Allen, 1997 ; Feldman, 1998 ; Pixa-Kettner, 1999). Des recherches documentent que les problèmes de comportement sont plus fréquents lorsque la mère ayant une DI souffre d'isolement social, lorsqu'elle subit un niveau élevé de stress parental (Aunos et al., 2008) ou lorsqu'elle présente un trouble de la santé mentale (McGraw et al., 2007). En fait, ces éléments contribueraient à l'adoption de styles parentaux moins positifs, plus hostiles et inconsistants/inefficaces, ce qui est associé à l'apparition plus fréquente de comportements problématiques chez l'enfant (Aunos et al., 2008). Une étude québécoise menée auprès de 50 mères relève que leurs enfants $(n=98)$ n'ont pas de comportements problématiques importants (Aunos et al., 2004). Par ailleurs, $5 \%$ d'entre eux ont reçu un diagnostic d'hyperactivité et $7 \%$ présentent un retard de développement ou une DI.

La DI du parent est considérée comme un facteur de risque important de négligence. La négligence manifestée par les mères ayant une DI proviendrait plus souvent « d'une ignorance et d'une incapacité à procurer les soins nécessaires, plutôt que d'un manque de volonté ou d'intérêt envers l'enfant » (Guay et al., 1997, p. 4). Selon ces derniers auteurs, la gravité de la négligence chez ces mères doit être comprise comme le résultat de plusieurs facteurs (stress, isolement social, pauvreté) qui, conjugués aux limitations cognitives, ont un effet cumulatif négatif sur la compétence parentale. Dans certains pays industrialisés, la proportion de ces enfants dans les systèmes de protection de l'enfance ${ }^{1}$ serait 60 fois plus élevée comparativement à ce qui serait attendu sur la base de leur prévalence au sein de la population générale (Booth et Booth, 2004). En outre, ces enfants feraient plus souvent l'objet d'un retrait de leur milieu familial (Booth et al., 2005 ; McConnell et al., 2011). Une étude canadienne révèle que $10 \%$ des dossiers ouverts annuellement pour une enquête relative à des mauvais traitements, soit environ 22 000, réfèrent à des situations d'enfants de parents ayant une DI (McConnell et al., 2011). Lorsqu'ils font l'objet de mesures de protection, de 40 à $60 \%$ de ces

${ }^{1}$ Cette terminologie est employée dans ce texte pour référer aux systèmes de protection hors Québec. En cas contraire, le terme protection de la jeunesse est utilisé. 
enfants sont retirés de leur famille d'origine (McConnell et Llewellyn, 2002 ; Collings et Llewellyn, 2012). Or, des auteurs font remarquer que les résultats des recherches disponibles sont biaisés puisqu'ils concernent, la plupart du temps, des participants déjà identifiés comme potentiellement abusifs ou négligents par les autorités de la protection de l'enfance (Aunos et al., 2003). Peu d'études portent sur la réalité des parents et/ou de leurs enfants qui vivent dans leur communauté et qui ne reçoivent pas de services d'établissements publics (Coppin, 2007 ; Hewitt, 2007).

\section{La réalité des parents}

Une multitude de facteurs ont une incidence sur les habiletés parentales des personnes présentant une DI (Feldman, 2002 ; Llewellyn et McConnell, 2010 ; Feldman et Aunos, 2011). Ces facteurs peuvent être d'ordre personnel, familial ou environnemental.

\subsection{Facteurs personnels}

Plusieurs personnes ayant une DI ont une histoire de vie marquée de stigmatisation, de ruptures de lien affectif et de mauvais traitements. Dans certains cas, elles n'ont pu côtoyer des modèles parentaux adéquats, soit parce qu'elles ont vécu en centre d'accueil, soit parce que leur vie familiale était chaotique ou dysfonctionnelle (Feldman, 2002 ; Coppin, 2007). Les résultats d'une étude menée auprès de 49 parents présentant une DI (30 femmes et 19 hommes) révèlent que $79,6 \%$ d'entre eux ont rapporté avoir fait l'objet d'abus ou de négligence pendant leur enfance (McGraw et al., 2007). Ces situations peuvent avoir limité l'acquisition de connaissances sur les besoins de base d'un enfant et sur la façon d'y répondre (Booth et Booth, 1998 ; Coppin, 2004 ; Llewellyn et McConnell, 2010).

Un nombre important d'études en DI ont porté sur la réalité des femmes. II en ressort que celles-ci ont davantage tendance à se conformer aux volontés des autres, ce qui les rend plus sujettes à l'exploitation (Tharinger et al., 1990). De plus, elles sont davantage à risque d'être victimes de violence sexuelle (Sobsey et Doe, 1991). En effet, les femmes présentant une DI sont de 4 à 10 fois plus à risque d'être victimes de violence sexuelle, de violence physique ou d'homicide par leur époux, leur conjoint ou leurs partenaires sexuels que toute autre femme présentant des incapacités (Wilson et Brewer, 1992 ; Sobsey, 2000). Des chercheurs constatent que les mères ayant une DI ont plus de difficulté à laisser un conjoint abusif en raison de ressources de soutien limitées (Aunos et Feldman, 2008 ; McGraw et al., 2010). Selon Booth et Booth (2000), l'incapacité de ces mères à se protéger elles-mêmes et à protéger leurs enfants contre les abus sexuels ou physiques commis par des conjoints est bien connue des systèmes de protection de l'enfance. Ce risque serait plus élevé lorsque la mère a elle-même été victime de sévices dans son histoire de vie (Khemka et Hickson, 2000 ; McGraw et al., 2007).

Comme les personnes ayant une DI ont un fonctionnement intellectuel et adaptatif limité, elles éprouvent des difficultés dans la réalisation des activités de la vie quotidienne (Juhel, 2000). Elles arrivent difficilement à bien évaluer les conséquences de leurs actions, à anticiper les évènements et à décoder les règles sociales (Éthier et al., 1999). Feldman (1998) remarque que plusieurs mères ayant une DI manquent de connaissances quant aux soins de base à 
donner à un nourrisson et quant aux besoins de l'enfant tout au long de son développement. Certaines d'entre elles éprouvent de la difficulté à interpréter les signaux émis par leur jeune enfant (Lynch et Bakley, 1989) ou à reconnaître les indices de maladie (Tymchuk et al., 1990a). Elles ont également plus de difficulté à prendre des décisions et à envisager des alternatives en situation de danger (Tymchuk et al., 1990b). Les parents ayant une DI auraient tendance à prendre au pied de la lettre les suggestions qu'ils reçoivent et à les appliquer de manière automatique (Whitman et Accardo, 1990). Par manque de connaissances et de jugement, ils ont plus de difficulté à assurer un environnement sécuritaire et stimulant à leur enfant (Feldman, 1994). Selon Feldman (1998), cette situation fait augmenter le niveau de risque d'abus et de négligence envers l'enfant et de difficultés d'adaptation ultérieures sur les plans comportemental, affectif et social.

Les mères présentant une DI se perçoivent généralement de façon négative, ont souvent une faible estime d'elles-mêmes et se considèrent moins compétentes que les autres mères (Keltner, 1992 ; Espe-Sherwindt et Crable, 1993). Bien que ce résultat ne soit pas concordant avec l'étude de Guay et al. (1997) sur 10 mères présentant une DI, certaines recherches mettent en lumière un niveau de stress parental plus élevé chez les mères ayant une DI (Booth et Booth, 1993 ; Feldman et al., 1997; Feldman et al., 2002), lequel tend à augmenter avec l'âge de l'enfant (Feldman et Walton-Allen, 1997). Cela peut s'expliquer par l'ampleur croissante des demandes des systèmes environnants (milieu de garde, école, etc.) et par les difficultés qu'éprouvent ces mères à communiquer avec leurs enfants plus âgés (Feldman et al., 1997). Leur vulnérabilité personnelle jumelée à des facteurs de risque, tels que l'isolement social, un statut socio-économique faible, des expériences difficiles pendant l'enfance et l'absence d'un conjoint soutenant, peut faire grimper leur niveau de stress à un seuil critique (Guay et al., 1997 ; Lalande et al., 2002). Pour Crittenden (2002), les mères qui vivent avec un haut niveau de stress ont davantage tendance à utiliser des stratégies de retrait, à ignorer leurs problèmes ou à déléguer leur autorité et leurs responsabilités parentales pour se protéger physiquement et psychologiquement.

Une étude australienne rapporte que les mères ayant une DI estiment avoir une santé physique et mentale moins bonne que celle des mères de même statut socioéconomique (Llewellyn et al., 2003). Une autre recherche menée auprès de 30 mères présentant une DI indique qu'environ $70 \%$ d'entre elles souffrent de dépression et/ou de troubles de l'anxiété (Feldman et al., 2002). Plusieurs manifestent une symptomatologie de dépression plus importante et plus sévère les mères sans DI (Tymchuk, 1994), ce qui affecterait leur capacité à apprécier leur maternité (Ehlers-Flint, 2002). Il est reconnu que la présence de troubles émotionnels affectant la santé mentale limite les aptitudes parentales (Tymchuk, 1994) et ce résultat est amplifié lorsque ces troubles sont jumelés à des limites cognitives. Par ailleurs, la dépression maternelle est associée à de multiples problèmes chez l'enfant (Beardslee et al., 1998 ; Goodman et Tully, 2006). Les enfants dont la mère souffre de dépression sont plus à risque de connaître des problèmes d'adaptation (Beardslee et al., 1998), de vivre une relation d'attachement insécurisante (Lyons-Ruth et al., 1990), de traverser des épisodes de dépression, de manifester des problèmes d'abus de substance et d'avoir des comportements d'opposition, 
des troubles des conduites et des problèmes de comportements (Bernard-Bonnin, 2004 ; Cummings et Kouros, 2009).

\subsection{Facteurs liés à l'environnement familial}

Des études indiquent que la majorité des familles dans lesquelles au moins un parent présente une DI vivent dans des conditions de précarité ; elles ont un revenu annuel peu élevé et un statut social faible (Tymchuk, 1992 ; Aunos et al., 2008). Plusieurs adultes présentant une DI sont exclus du marché du travail (Emerson, 2007 ; Picard et al., 2011) et, s'ils travaillent, ils occupent des emplois précaires (Aunos, 2004 ; Aunos et Feldman, 2010). L'impact de leur faible revenu est souvent aggravé par le fait qu'ils n'ont pas les compétences nécessaires pour faire une gestion serrée du budget familial (Ray et al., 1994 ; Desmet, 2005).

Tel que l'indique certains auteurs, ce n'est pas la pauvreté en soi qui génère des problèmes dans la vie des personnes présentant une DI, mais bien l'exposition à une diversité de facteurs de risque découlant de leur faible statut socioéconomique et de l'exclusion sociale dont elles font l'objet (Emerson, 2011 ; Hyppolite, 2012 ; Emerson et Brigham, 2013). En effet, les parents présentant une DI n'ont pas toujours accès aux mêmes ressources que les autres parents, telles que des conditions de logement adéquates, la stabilité d'emploi, une bonne santé et des soins et services de qualité (McConnell, 2008). Vroman (2008) constate qu'un nombre important de parents ayant une DI vivent, sur les plans du logement, des finances, de l'hygiène et de l'alimentation, dans des conditions peu propices au développement d'un enfant.

Taylor et al. (2010) relèvent une fréquentation scolaire plus courte chez les enfants de parent ayant une DI; les chercheurs émettent l'hypothèse que cette situation serait en partie attribuable aux croyances des parents à l'égard des avantages de la fréquentation scolaire. D'autres recherches rapportent, au contraire, que ces parents attribuent une grande importance au cheminement scolaire (Strike et McConnell, 2002). Lorsqu'ils reçoivent les mesures de soutien et d'accompagnement adéquates, leurs enfants jouissent habituellement d'une meilleure intégration communautaire et d'une fréquentation scolaire plus assidue (Tarleton et al., 2006).

\subsection{Facteurs liés à l'environnement social}

Les personnes qui présentent une DI ont généralement davantage de difficulté à établir des relations sociales significatives et, plus particulièrement, des relations qui rehaussent leur estime de soi ou qui peuvent être des sources de soutien (Edgerton, 2001). La plupart des mères ayant une DI ont un réseau social restreint, constitué principalement de membres de la famille et d'intervenants (Llewellyn et al., 1999 ; Llewellyn et McConnell 2002 ; Aunos, 2004 ; St-Amand et al., 2010a). Des études québécoises, américaines et australiennes relèvent que le quart de ces mères n'auraient aucun ami et que, pour les autres, les amis constitueraient de $9 \%$ à $21 \%$ de leur réseau social (Lewellyn et al., 1999 ; Ehlers-Flint, 2002 ; Llewellyn et McConnell, 2002 ; Aunos et al., 2008). Aussi, elles sont peu nombreuses à participer à des activités dans leur communauté d'appartenance (Aunos et al., 2004 ; St-Amand et al., 2010b). Or, plusieurs chercheurs mettent en lumière que ce n'est pas tant le nombre de personnes constituant ce 
réseau de soutien qui importe, mais bien, la qualité du soutien offert (Espe-Sherwindt et Crable, 1993 ; Feldman et al., 2002 ; Aunos et al., 2004).

Des études québécoises récentes menées auprès de mères ayant une DI mettent en lumière un taux de satisfaction généralement élevé à l'égard de l'aide offerte (Aunos et al., 2004 ; StAmand et al., 2010a). Cependant, certaines d'entre elles n'apprécient pas que des membres de leur réseau remettent en question leur aptitude à élever leur enfant, critiquent leurs habiletés parentales et tentent de prendre le contrôle de leur vie (Booth et Booth, 1995 ; Llewellyn, 1995 ; Ehlers-Flint, 2002 ; Mayes et al., 2006 ; St-Amand et al., 2010a). Pour certaines mères, ces attitudes affectent leur estime de soi et nuisent à leur confiance en leurs capacités (Llewellyn, 1995 ; Llewellyn et al., 1999). Or, une appréciation positive du soutien social est associée à un niveau de bien-être psychologique plus élevé et à de meilleures habiletés parentales (Llewellyn et al., 1999 ; Aunos et al., 2008). Celle-ci est donc liée au bien-être des enfants, par son effet direct sur les pratiques parentales (Wade et al., 2011).

Pour répondre spécifiquement aux besoins du nombre croissant de parents présentant une DI, certains services ont été développés au Québec et ailleurs. Cependant leur mise en place comporte des défis importants qui sont abordés à la section suivante.

\section{Défis et enjeux dans l'offre de services psychosociaux}

Plusieurs parents ayant une DI ont des besoins personnels, affectifs et sociaux auxquels il faut apporter une réponse avant d'envisager des actions pour développer leurs compétences parentales. Or, ces besoins ne sont pas toujours considérés dans l'offre de services (Aunos et al., 2010a); les mesures de soutien et d'éducation demeurent insuffisantes (Llewellyn et Bridgen, 1995 ; McBrien et Power, 2002 ; Aunos et al., 2010b).

Tarleton et Ward (2007) constatent que des services généraux, comme les cours prénataux, ne sont pas facilement accessibles pour les parents ayant une DI ; ils s'y sentent mis à l'écart. En 1985, Gilhool et Gran identifiaient trois facteurs pouvant justifier le manque de services destinés spécifiquement à ces parents soit : l'absence de ressources, la présomption que les personnes ayant une DI ne peuvent pas apprendre et la croyance qu'il est préférable de placer l'enfant plutôt que d'offrir des services pédagogiques à ces parents. Or, ces représentations sont toujours bien présentes, ce que rapportent des écrits plus récents à ce sujet (McConnell, 2008 ; Llewellyn et al., 2010).

Lorsque les services existent, les moyens utilisés ne tiennent pas toujours compte des difficultés des parents et ne sont pas adaptés à leur réalité (Aunos et Feldman, 2007). Deux études connues évaluent le taux de décrochage des services destinés aux familles où au moins un parent présente une DI à environ 40 \% par année (Ray et al., 1994 ; Wade et al., 2007).

Parmi les facteurs explicatifs du faible pouvoir attractif de ses services, l'attitude des intervenants serait en cause. Selon Marx (2008), les inquiétudes des intervenants à l'égard de la sécurité ou du bien-être des enfants contribuent à mettre de la pression sur les épaules des parents. Certaines familles, habitées par un fort sentiment d'impuissance, un manque de 
confiance en leurs ressources personnelles et une perte de l'espoir d'arriver un jour à s'occuper adéquatement de leurs enfants, iraient même jusqu'à « l'abandon de ces enfants "phagocytés" par les professionnels et fondent le projet de faire un autre enfant qui échapperait, celui-là, aux services sociaux » (Marx, 2008, p. 49). D'ailleurs certains parents déplorent l'intransigeance et le manque de confiance des intervenants. Selon Starke (2010), de nombreux parents ayant une DI ne se sentent pas à l'aise dans leurs interactions avec ceux-ci. Ils vivent leurs visites comme intrusives et servant à les juger plutôt qu'à les aider. Leurs difficultés à occuper adéquatement leur rôle parental s'en trouvent alors aggravées par leur sentiment d'être toujours surveillés par les professionnels et, parfois, par des membres de leur famille. Cette situation contribue à amplifier leur sentiment de ne pas être à la hauteur (Desmet, 2005 ; Leroux et Scelles, 2007). Cette réalité est susceptible d'engendrer un cercle vicieux dans lequel, d'une part, les parents ne font aucune demande d'aide ou rejettent les services proposés afin de démontrer qu'ils sont capables d'élever leur enfant seuls et, d'autre part, ils sont accusés de ne pas comprendre la complexité de l'éducation d'un enfant parce qu'ils déclinent les services offerts (McConnell et Llewellyn, 2000 ; Aunos et Feldman, 2008). Cette appréhension serait encore plus marquée lorsqu'il s'agit d'intervenants dont le mandat est d'assurer la protection des enfants (Tarleton et al., 2006). Desmet (2005, p. 12) va jusqu'à dire que les parents vivant avec une DI sont « constamment placé[s] sous le regard des autres, parents, intervenants et membres de la société, qui évaluent, scrutent, mesurent [leurs] compétences » de parents et « qui risquent, à la moindre défaillance, de [les] déclarer inaptes à élever [leurs] enfants ».

Plusieurs chercheurs ont analysé les rapports d'évaluation psychosociale ayant mené au retrait de l'enfant de son milieu familial dans différents pays industrialisés (Hertz, 1979; Hayman, 1990 ; Levesque, 1996 ; Glaun et Brown, 1999 ; McConnell et Llewellyn, 2000 ; Llewellyn et al., 2003 ; Booth et al., 2005 ; Mayes et Llewellyn, 2009 ; Lightfoot et al., 2010). Ils concluent que les décisions rendues sont davantage conditionnées par les préjugés négatifs à l'endroit des parents que par une démonstration réelle de maltraitance puisque les allégations sur le manque de compétence de ces parents ont souvent été reconnues non fondées. Selon Levesque (1996), lorsqu'un ou les deux parents présentent une DI, il y a perte de la garde de l'enfant sur la base de motifs qui, pour d'autres parents, ne seraient pas suffisants pour conduire au placement. Les jugements à l'endroit de ces parents seraient plus sévères qu'à l'endroit de ceux qui vivent des problèmes de santé mentale ou de toxicomanie (Booth et al., 2005 ; Cleaver et Nicholson, 2007 ; Mayes et Llewellyn, 2009). Les parents ayant une DI seraient souvent perçus comme des «enfants » eux-mêmes; ils ne pourraient donc pas assumer la responsabilité d'un être vulnérable (Hayman, 1990 ; Llewellyn et McConnell, 2010). Certains intervenants estiment que leurs difficultés sont insurmontables et permanentes (Swain et Cameron, 2003; Booth et Booth, 2004). D'autres les croient incapables d'acquérir les compétences nécessaires pour répondre aux besoins évolutifs de leurs enfants (McConnell et Sigurjónsdóttir, 2010).

Les intervenants sociaux se sentent généralement démunis, invoquant plusieurs facteurs de stress propres à leurs conditions de travail, tels que des connaissances et une expertise limitées, une importante charge de travail, l'ampleur des difficultés des clients et les défis liés à la collaboration avec les partenaires (Clayton et al., 2008). Aussi, la coopération entre les parents et les professionnels se heurte souvent à des préjugés, et parfois à un manque de 
compréhension et d'ouverture à la réalité de l'autre (McConnell, 2008). Ces éléments nuisent à l'acceptation des services par les parents eux-mêmes (Aunos et al., 2010a).

Plusieurs professionnels en protection de l'enfance ne possèdent pas l'expertise pour l'intervention auprès des parents ayant une DI (Aunos et Feldman, 2007 ; McConnell et al., 2011). Ils rapportent se sentir très mal outillés pour évaluer le niveau de risque au sein de ces familles. Ils ont donc tendance à diriger ces parents vers des organismes communautaires (OC) pour la famille, même s'ils savent que leurs services peuvent être mal adaptés à leurs besoins particuliers (McConnell et al., 2006). Des recherches nord-américaines indiquent que les parents ayant une DI, dont l'enfant fait l'objet d'un signalement pour soupçons d'abus ou de négligence, bénéficient de peu de services visant à leur permettre d'appliquer les mesures suggérées à la Cour pour conserver la garde de l'enfant (Hertz, 1979 ; McConnell et Sigurjónsdóttir, 2010).

Dans le cadre d'une étude européenne, des parents ayant une DI rapportent se sentir seuls et laissés pour compte lorsque la garde de leur enfant est abordée en Cour, un lieu qui leur semble impersonnel, injuste et débilitant (Hunt et al., 1999). D'autres ne se sentent ni soutenus ni entendus, même par l'avocat qui les représente (McConnell et Sigurjónsdóttir, 2010). Ils ont l'impression d'occuper un rôle de spectateur impuissant dans une démarche complexe et intimidante regroupant des experts qui décident de leur sort et du futur de leur enfant. Certains ont l'impression que les travailleurs sociaux interviennent dans leur dos et qu'ils gardent secrètes des informations qu'ils seraient en droit d'obtenir (Wade et al., 2007 ; McConnell et Sigurjónsdóttir, 2010). Lorsque la question de la garde est discutée, certains parents ressentent de la colère et de l'indignation lorsqu'ils constatent que leurs erreurs, qu'ils considèrent bien minimes, sont évoquées pour justifier leur présumée incompétence (Booth et Booth, 2005). D'autres vivent un profond sentiment de désespoir et de défaite, estimant que les jugements des autorités dépeignent leur réalité de manière distordue, en plus de ne pas donner de crédit à leurs forces et à leurs progrès (McConnell et Sigurjónsdóttir, 2010). Booth et al. (2006) soulignent que des intervenants en protection de l'enfance perçoivent chez ces parents un manque de coopération et de volonté à conserver la garde de leur enfant. Selon ces auteurs, il s'agit d'une représentation souvent erronée, attribuable aux limitations intellectuelles des parents et à leur faible estime de soi. En effet, certains sont jugés peu fiables puisqu'ils ne respectent pas leurs engagements et leurs rendez-vous (Booth et al., 2006), alors que cette situation peut être attribuable au fait qu'ils ont de la difficulté à comprendre des concepts abstraits, tels que l'heure et l'organisation des systèmes de transport en commun (Aunos et Feldman, 2010 ; McConnell et al., 2011). Des parents ont développé une anxiété paralysante provoquée par un sentiment de peur généralisée à l'endroit de tout professionnel des services de protection de l'enfance (McConnell et Llewellyn, 2000). Aussi, lorsqu'ils sont questionnés à la Cour, certains acquiescent à ce qui leur est dit, sans nécessairement en comprendre la signification (Booth et al., 2006). II s'agit aussi d'une réaction observée chez un grand nombre de personnes ayant une DI lorsqu'ils sont interrogés (Guillemette et Boisvert, 2003 ; Julien-Gauthier et al., 2009).

Somme toute, il ressort que plusieurs parents présentant une DI font face à des préjugés ou n'obtiennent pas le soutien adéquat, ce qui nuit au respect de leurs droits fondamentaux (Booth et Booth, 1993; Feldman, 2002) et au 
droit de l'enfant de vivre dans un milieu stable, sécuritaire et stimulant (ministère de la Santé et des Services sociaux [MSSS], 2007).

\section{Recommandations à l'égard de l'intervention et des services}

Plusieurs parents ayant une DI peuvent développer de meilleures habiletés parentales lorsqu'ils reçoivent les services et le soutien appropriés (Feldman, 1994 ; Feldman, 2010 ; Aunos, 2000 ; Aunos et al., 2003 ; Wade et al., 2007). En effet, ils peuvent apprendre: à développer des interactions positives avec leur enfant ; à améliorer leurs habiletés de résolution de problèmes ; à développer des savoir-faire utiles à la réalisation des activités de leur vie quotidienne ; à assurer la sécurité de leur enfant et à identifier les approches appropriées à une meilleure gestion des comportements (Wade et al., 2008). Généralement, ces objectifs peuvent être atteints lorsqu'ils reçoivent $\mathrm{du}$ soutien et bénéficient d'interventions éducatives et psychosociales adaptées à leurs besoins.

\subsection{La nature des actions}

Aunos (2006) recommande que tout parent (actuel ou futur) ayant une DI soit identifié le plus tôt possible et que sa situation familiale fasse l'objet d'une évaluation exhaustive visant à bien cerner ses forces et ses besoins. Celle-ci doit tenir compte de ses habiletés parentales et de ses compétences connexes (habiletés sociales, aptitude à résoudre des problèmes, etc.). Elle doit aussi couvrir les facteurs personnels, familiaux et environnementaux pouvant influencer positivement ou négativement les compétences parentales et le développement de l'enfant (Aunos et Feldman, 2007 ; Aunos et Feldman, 2010). L'évaluation doit s'appuyer sur des observations directes et in situ. Pour mener à bien cette action précoce, l'évaluateur doit détenir une expertise qui lui permet de conduire une démarche objective, exempte de biais et inspirée par les plus récentes connaissances en matière de stratégies d'interventions éducatives et psychosociales efficaces (Feldman, 1994 ; Feldman et Aunos, 2011).

Lorsque l'évaluation est complétée, des objectifs doivent être déterminés avec le parent et une offre de services impliquant différents intervenants doit être proposée. À ce sujet, les interventions réalisées dans un esprit d'empowerment de la famille sont généralement plus efficaces et mieux reçues par les parents (Webster-Stratton, 1997 ; Webster-Stratton, 1998 ; Aunos, 2004 ; Wade et al., 2007). Une telle approche suppose que l'intervenant témoigne aux parents sa reconnaissance de leurs capacités et son ouverture à l'égard des solutions qu'ils mettent de l'avant. Lorsque les interventions sont flexibles, modulées selon les besoins évolutifs des familles et offertes à long terme, elles sont plus susceptibles d'atteindre leurs objectifs (Llewellyn, 1995 ; Llewellyn et McConnell, 2002 ; Wade et al., 2007). Aussi, ces interventions doivent être adaptées au style d'apprentissage des parents et se réaliser dans un environnement familier, de façon à favoriser le transfert des nouveaux acquis (Feldman, 1998 ; Feldman, 2010 ; Collings et Llewellyn, 2012). Elles doivent également miser sur un suivi à long terme et sur des techniques d'intervention favorisant le transfert et la généralisation (Tymchuk, 1999 ; Llewellyn et al., 2002). Il importe également que les stéréotypes et les préjugés des intervenants impliqués soient mis à jour (Jöreskog et Starke, 2013), d'où l'importance qu'ils soient accompagnés dans leur pratique et outillés en termes d'information et de formation. Toutes ces recommandations 
ont été prises en compte dans le développement d'un projet pilote développé dans un CRDITED de la région de Montréal, Québec. Les retombées de son implantation peuvent être appréciées dans l'article de MacLean et Aunos (2013).

\subsection{La préparation des intervenants}

Considérant les défis de l'intervention auprès de ces parents, Desmet (2005) insiste sur l'importance de mettre en place des mesures de soutien et d'encadrement pour les cliniciens. Les « comités parentalité » développés dans différents CRDITED du Québec sont un exemple de telles mesures. Ces comités, qui sont constitués de conseillers à l'intervention, d'intervenants expérimentés et de professionnels spécialisés en parentalité et DI, en développement de l'enfant et en prévention de la négligence, visent à créer un lieu d'échange et de partage. Ils offrent aussi de la formation à travers des lectures et des conférences. Dans l'étude de Desmet (2005), un intervenant mentionne que sa participation à un tel comité lui a permis de mieux connaître les ressources existantes sur le territoire. Un second rapporte que d'y être impliqué « l'a amené à modifier sa perception » de la parentalité des personnes présentant une DI « et lui a offert des arguments pour répondre aux intervenants qui s'opposent à celle-ci » (p. 53).

Les intervenants impliqués doivent également connaître les bases de l'intervention éducative et psychosociale en DI (Aunos et Feldman, 2007 ; Aunos et Feldman, 2010). Pour favoriser pleinement les apprentissages des parents, les interventions doivent porter non seulement sur les connaissances, mais aussi sur des habiletés concrètes. Dans ce but, les intervenants doivent maîtriser différentes techniques comportementales pour favoriser l'apprentissage de nouvelles habiletés, telles que le modelage, les instructions verbales simplifiées, la rétroaction et le renforcement positif des comportements cibles (Hur, 1997 ; Aunos, 2000 ; Feldman, 2004 ; Feldman, 2010). Ils doivent aussi savoir adapter ou créer du matériel visuel pédagogique pour pallier les difficultés de compréhension et de lecture de certains parents.

Plusieurs parents ayant une DI reconnaissent leur besoin de soutien et sont ouverts à l'idée de collaborer avec les professionnels, à condition d'être considérés comme des adultes (Booth et Booth, 2004 ; Leroux et Scelles, 2007 ; Starke, 2010). Selon Ray et al. (1994), les intervenants qui établissent les contacts plus positifs avec les parents ayant une DI sont ceux qui respectent leur rythme, qui ne les jugent pas, qui sont flexibles et qui font preuve de compassion. Ces intervenants doivent aussi adopter une attitude positive et respectueuse et exprimer leur foi en leur capacité à être ou à devenir de bons parents (Tarleton et Ward, 2007). Une telle attitude amènerait les parents à développer une perception plus positive de l'intervention (Aunos et al., 2004) et à amorcer une dynamique de «prophéties autoréalisatrices » (Coppin, 2001). II s'agit aussi de respecter les demandes des parents, leurs croyances et leurs valeurs: un aspect essentiel dans la création d'une relation de confiance. Celle-ci est incontournable pour assurer l'ouverture du parent vis-à-vis des apprentissages proposés dans l'exercice de son rôle parental (Espe-Sherwindt et Crable, 1993 ; Guinea, 2001).

Idéalement, chaque parent présentant une DI devrait s'engager dans l'apprentissage des habiletés parentales nécessaires au développement de son enfant. Aunos (2006) suggère de développer également des services résidentiels pour les familles ayant besoin d'interventions 
individualisées plus intenses et de prévoir des mécanismes de collaboration avec les différents établissements offrant des services à ces parents (CSSS, centres jeunesse, centres de la petite enfance, écoles, O.C., etc.). Un tel partenariat pourrait être facilité par l'adoption d'une politique conjointe de services. Selon Desmet (2005), le partenariat entre les établissements impliqués fait bien souvent une différence dans le bien-être des familles de parents ayant une DI en établissant des balises pour l'intervention de chacun et en développant une offre plus cohérente de services. II est aussi recommandé de miser sur la création d'un modèle de parentalité flexible et adapté dans lequel le parent occupe un rôle valorisant et à la hauteur de ses aspirations et l'enfant, quel que soit son âge, trouve une place au sein de sa famille où il se sent entendu, compris et aimé (Gauthy, 2008). Selon Aunos (2006), il s'agit de réfléchir à une orientation de services qui réponde à un besoin psychosocial et humain.

\section{DISCUSSION}

Même si la recherche évolue rapidement, les connaissances sur la parentalité des personnes ayant une DI comportent toujours certaines limites. D'abord, peu d'études se rapportent au contexte québécois (Aunos, 2004 ; Desmet, 2012). De plus, les recherches sont souvent réalisées auprès de petits échantillons, souvent non représentatifs, constitués de parents ayant demandé ou obtenu de l'aide des services sociaux ou de la protection de l'enfance (Booth et Booth, 1993 ; McConnell et al., 2011). Par ailleurs, l'information disponible provient presque uniquement des mères (Aunos, 2004 ; Mayes et Sigurjónsdóttir, 2010). Or, un père est présent dans environ 60 \% des familles où un parent a une DI (Feldman et al., 1997 ; Guay et al., 1997).

\section{CONCLUSION}

Dans l'état actuel des connaissances, il s'avère ardu de dresser un portrait exhaustif de la parentalité des personnes présentant une DI au Québec et ailleurs. Selon Coppin (2007) et Chatroussat (2011), les limites à la fiabilité et à l'ampleur des connaissances actuelles contribuent à faire perdurer certains processus de stigmatisation et de préjugés à l'égard des parents ayant une DI. Pourtant, mieux connaître leurs besoins et y répondre adéquatement est fondamental pour une société qui prône la justice sociale et l'équité pour tous.

Beaucoup d'efforts doivent être investis pour mieux outiller les professionnels qui côtoient des parents présentant une DI. Une réflexion s'impose aussi quant à leur préparation à intervenir auprès des personnes ayant une DI. À titre d'exemple, la formation de premier cycle en travail social offerte dans plusieurs universités québécoises ne contient aucun cours spécifique à l'intervention en DI. Or, les futurs travailleurs sociaux auront certainement à côtoyer des parents ayant une DI ou une LI à travers leur parcours professionnel.

Plusieurs parents vivant avec une LI, aux limites du diagnostic, font partie d'une «majorité invisible », inconnue du système de santé et des services sociaux. Pour éviter que leurs enfants fassent davantage l'objet de placement (McConnell et al., 2002), il faut s'assurer de la mise en place de mesures de soutien adaptées aux besoins des parents. II serait également pertinent d'analyser les représentations sociales des intervenants de la protection de la jeunesse du Québec à l'égard de la parentalité de ces adultes, ainsi que l'effet de ces représentations sur leurs pratiques professionnelles. Une autre étude pourrait aborder la perception de ces parents 
québécois en ce qui a trait aux services offerts. La considération de leur perspective paraît cruciale dans l'élaboration de recommandations plus justes destinées aux décideurs des organisations de services. Elle peut également encourager une réflexion sur le soutien nécessaire à l'exercice effectif de leurs droits parentaux, tout comme le droit des enfants de vivre dans un milieu sécuritaire, bienveillant et stimulant.

MILOT, Élise

Doctorante en service social École de service social Université Laval

TURCOTTE, Daniel Professeur titulaire École de service social Université Laval TÉTREAULT, Sylvie Professeure titulaire Faculté de médecine Université Laval

\section{BIBLIOGRAPHIE}

Aunos, M. (2000). «Les programmes de formation aux habiletés parentales pour des adultes présentant une déficience intellectuelle », La revue internationale de l'éducation familiale, vol. $4, n^{0} 2$, p. 59-75.

Aunos, M. (2004). Études de variables parentales sur le comportement des enfants de mères recevant des services de centres de réadaptation: Études comparatives et exploratoires (thèse de doctorat), Montréal, Université du Québec à Montréal.

Aunos, M. (2006). "Quand vie affective et sexualité se traduisent en parentalité » dans $\mathrm{H}$. Gascon, D. Boisvert, M.-C. Haelewyck, J.-R. Poulin et J.-J. Detraux, Déficience intellectuelle : savoirs et perspectives d'action, tome 2 : Formation, interventions, adaptation et soutien social, Québec, Presses Interuniversitaires, p. 339-347.

Aunos, M., et M. Feldman (2002). «Attitudes towards sexuality, sterilization and parenting rights of persons with intellectual disabilities », Journal of Applied Research in Intellectual Disabilities, vol. 15, $\mathrm{n}^{\circ} 4$, p. 285-296.

Aunos, M., et M. Feldman (2007). «Parenting by people with intellectual disabilities » dans I. Brown et M. Percy (dir.), A Comprehensive Guide to Intellectual and Developmental Disabilities, Baltimore, Paul H. Brookes publishing, p. 593-603.

Aunos, M., et M. Feldman (2008). «There's no place like home: The child's right to family » dans T. O'Neil et D. Zinga, Children's Rights: Multidisciplinary Approaches to Participation and Protection, Toronto, University of Toronto Press, p. 137-162. 
Aunos, M. et M. Feldman (2010). «Assessing parenting capacity in parents with intellectual disabilities » dans $\mathrm{C}$. Chamberland, S. Léveillé et $\mathrm{N}$. Trocmé, Enfants à protéger, parents à aider : deux univers à rapprocher, Québec, Presses de l'Université du Québec, p. 223-240.

Aunos, M., M. Feldman et G. Goupil (2008). «Mothering with intellectual disabilities: Relationship between social support, health and well-being, parenting and child behavioral outcomes», Journal of Applied Research in Intellectual Disabilities, vol. 21, n 4, p. 320-330.

Aunos, M., G. Goupil et M. Feldman (2003). «Les mères présentant une déficience intellectuelle : Revue de littérature », Handicap - Revue de sciences humaines et sociales, $\mathrm{n}^{\circ} 97$, p. 32-52.

Aunos, M., G. Goupil et M. Feldman (2004). « Mothers with intellectual disabilities who do or do not have custody of their children », Journal on Developmental Disabilities, vol. 10, $\mathrm{n}^{\circ} 2, \mathrm{p}$. 65-80.

Aunos, M., G. Goupil et K. St-Amand (2010a). «Qualité de vie et préparation à la vie parentale des personnes qui présentent une déficience intellectuelle », dans M.-C. Haelewyck et $\mathrm{H}$. Gascon (dir.), Adolescence et retard mental, Bruxelles, De Boeck, p. 223-231.

Aunos, M., et L. Pacheco (2013). « Changing perspective: Workers' perceptions of inter-agency collaboration with parents with an intellectual disability », Journal of Public Child Welfare, vol. $7 n^{\circ} 5$, p. $658-674$.

Aunos, M., L. Pacheco et K. Moxness (2010b). «Turning rights into realities in Québec, Canada », dans G. Llewellyn, R. Traustadóttir, D. McConnell et H. B. Sigurjónsdóttir (dir.), Parents with Intellectual Disabilities: Past, Present and Futures, West Sussex, WileyBlackwell, p. 189-204.

Azar, S.T., M.C. Maggi et S.N. Proctor (2013). «Practices changes in the child protection system to address the needs of parents with cognitive disabilities », Journal of Public Child Welfare, vol. $7, n^{\circ} 5$, p. $610-632$.

Beardslee, W.R., E.M. Versage et T.R.G. Gladstone (1998). « Children of affectively ill parents: A review of the past 10 years ", Journal of the American Academy of Child and Adolescent Psychiatry, vol. 37, $\mathrm{n}^{\circ} 11$, p. 1134-1141.

Bernard-Bonnin, A.-C. (2004). «La dépression de la mère et le développement de l'enfant », Pediatric Child Health, vol. 9, nº 8, p. 589-598.

Booth, T., et W. Booth (1993). « Parenting with learning difficulties: Lessons for practitioners », British Journal of Social Work, vol. 23, $\mathrm{n}^{\circ} 5$, p. 459-480.

Booth, T., et W. Booth (1995). « Unto us a child is born: The trials and rewards of parenthood for people with learning difficulties », Australia and New Zealand Journal of Developmental Disabilities, vol. 20, $\mathrm{n}^{\circ} 1$, p. 25-39.

Booth, T., et W. Booth (1998). Growing up with parents who have learning difficulties, Londres et New York, Routledge.

Booth, T., et W. Booth (2000). « Against the odds: growing up with parents who have learning difficulties », Mental Retardation, vol. 38, $\mathrm{n}^{0} 1$, p. 1-14. 
Booth, T., et W. Booth (2004). « Brief research report: Findings from a court study of care proceedings involving parents with intellectual disabilities », Journal of Policy in Intellectual Disabilities, vol. 1, n ${ }^{\text {os }}$ 3-4, p. 179-181.

Booth, T., et W. Booth (2005). « Parents with learning difficulties in the child protection system: Experiences and perspectives », Journal of Intellectual Disabilities, vol. 9, n² 2, p. 109-129.

Booth, T., W. Booth et D. McConnell (2005). «The prevalence and outcomes of care proceedings involving parents with learning difficulties in the family courts », Journal of Applied Research in Intellectual Disabilities, vol. 18, $\mathrm{n}^{\circ} 1, \mathrm{p}$. 7-17.

Booth, T., D. McConnell et W. Booth (2006). « Temporal discrimination and parents with learning difficulties in the child protection system », British Journal of Social Work, vol. 36, $\mathrm{n}^{\circ}$ 6, p. 9971015.

Chatroussat, S. (2011). «La parentalité des personnes déficientes intellectuelles: Entre stigmatisation et intégration », Dialogue: Recherches sur le couple et la famille, vol. 194, $\mathrm{n}^{\circ} 4$, p. 57-67.

Clayton, O., A. Chester, R. Mildon et J.M. Matthews (2008). «Practionners who work with parents with intellectual disability: Stress, coping and training needs », Journal of Applied Research in Intellectual Disabilities, vol. 21, p. 367-376.

Cleaver, H., et D. Nicholson (2007). Parental Leaning Disability and Children's Needs: Family Experiences and Effective Practice, Londres, Jessica Kingsley Publishers.

Collings, S., et G. Llewellyn (2012). « Children of parents with intellectual disability: Facing poor outcomes or faring okay? », Journal of Intellectual \& Developmental Disability, vol. 37, $\mathrm{n}^{\circ}$ 1, p. 65-82.

Coppin, B. (2001). «Déficience intellectuelle et parentalité », Revue francophone de la déficience intellectuelle, vol. 12, $\mathrm{n}^{0} 2$, p. 243-257.

Coppin, B. (2004). « Être parent avec une déficience intellectuelle », Pratiques psychologiques, vol. 10, p. 25-38.

Coppin, B. (2007). «Être parent et en situation de handicap: Des idées reçues à quelques réalités », Reliance: Revue des situations de handicap, de l'éducation et des sociétés, vol. $26, n^{\circ} 4$, p. 88-96.

Crittenden, P.M. (2002). " Family and dyadic patterns of functioning in maltreating families », dans K. Browne, C. Davies et P. Stratton (dir.), Early Prediction and Prevention of Child Abuse, New York, John Wiley \& Sons, p. 161-189.

Cummings, E.M., et C.D. Kouros (2009). «La dépression de la mère et sa relation avec le développement et l'adaptation des enfants », Encyclopédie sur le développement des jeunes enfants, Montréal, Centre d'excellence pour le développement des jeunes enfants et Réseau stratégique de connaissances sur le développement des jeunes enfants. Disponible en ligne $<$ http://www.enfant-encyclopedie.com/pages/pdf/cummings-kourosfrxp.pdf>. 
Desmet, S. (2005). Les situations de parentalité au centre de services en déficience intellectuelle Mauricie/Centre du Québec, Trois-Rivières, Centre de services en déficience intellectuelle de la Mauricie et du Centre-du-Québec.

Desmet, S. (2012). La sensibilité des mères présentant une déficience intellectuelle (thèse de doctorat), Trois-Rivières, Université du Québec à Trois-Rivières.

Edgerton, R.B. (2001). «The hidden majority of individuals with mental retardation and developmental disabilities », dans A.J. Tymchuk, K.C. Lakin et R. Luckasson (dir.), The Forgotten Generation: The Status and Challenges of Adults with Mild Cognitive Limitations, Baltimore, Paul H. Brookes, p. 3-20.

Ehlers-Flint, M.L. (2002). «Parenting perceptions and social supports of mothers with cognitive disabilities », Sexuality and Disability, vol. 20, $n^{\circ} 1$, p. 29-51.

Emerson, E. (2007). « Poverty and people with intellectual disabilities », Mental Retardation and Developmental Disabilities Research Reviews, vol. 13, n² 2, p. 107-113.

Emerson, E. (2011). " Health status and health risks of the "hidden majority" of adults with intellectual disability », Intellectual and Developmental Disabilities, vol. 49, n 3, p. 155-165.

Emerson, E., et P. Brigham (2013). « Health behaviours and mental health status of parents with intellectual disabilities: Cross sectional study », Public Health, vol. 127, n 12, p. 1111-1116.

Espe-Sherwindt, M., et S. Crable (1993). «Parents with mental retardation: Moving beyond the myths », Topics in Early Childhood Special Education, vol. 13, n², p. 154-174.

Éthier, L. S., C. Biron, M. Boutet et C. Rivest (1999). « Les compétences parentales chez les personnes présentant des incapacités intellectuelles: État de la question », Revue francophone de la déficience intellectuelle, vol. 10, $\mathrm{n}^{\circ}$ 2, p. 109-121.

Feldman, M. (1994). «Parenting education for parents with intellectual disabilities: A review of outcome studies », Research in Developmental Disabilities, vol. 15, n 4, p. 299-331.

Feldman, M. (1998). «Preventing child neglect: Child-care training for parents with intellectual disabilities », Infants and Young Children, vol. 11, $\mathrm{n}^{\circ} 2$, p. 1-11.

Feldman, M. (2002). «Parents with intellectual disabilities: Impediments and supports », dans D. Richardson (dir.), Ethical Dilemmas: Sexuality and Developmental Disability, Kingston (NY), NADD Press, p. 255-292.

Feldman, M. (2004). «Self-directed learning of child-care skills by parents with intellectual disabilities », Infants and Young Children today, vol.17, $\mathrm{n}^{\circ} 1$, p. 17-31.

Feldman, M. (2010). « Parenting education programs », dans G. Llewellyn, R. Traustadóttir, D. McConnell et H. B. Sigurjónsdóttir (dir.), Parents with Intellectual Disabilities: Past, Present and Futures, West Sussex, John Wiley \& Sons, p. 121-136.

Feldman, M., et M. Aunos (2011). Comprehensive Competence-based Parenting Assessment for Parents with Learning Difficulties and Their Children, Kingston (NY), NADD Press. 
Feldman, M., L. Case, F. Towns et J. Betel (1985). «Parent education project I: The development and nurturance of children of mentally retarded parents », American Journal of Mental Deficiency, vol. 90, $\mathrm{n}^{\circ} 3$, p. 253-258.

Feldman, M., M. Leger et N. Walton-Allen (1997). "Stress in mothers with intellectual disabilities », Journal of Child and Family Studies, vol. 6, $n^{\circ} 4$, p. 471-486.

Feldman, M., J. Varghese, J. Ramsay et D. Rajska (2002). « Relationships between social support, stress and mother-child interactions in mothers with intellectual disabilities », Journal of Applied Research in Intellectual Disabilities, vol. 15, p. 314-323.

Feldman, M., et N. Walton-Allen (1997). « Effects of maternal mental retardation and poverty on intellectual academic and behavioral status of school-age children », American Journal on Mental Retardation, vol. 101, $\mathrm{n}^{\circ} 4$, p. 352-364.

Gauthy, L. (2008). « De l'importance du réseau », dans M. Mercier et G. Brazier, Parentalité des personnes déficientes mentales, Namur, Presses universitaires de Namur, p. 23-26.

Gilhool, T.K., et J.A. Gran (1985). « Legal rights of disabled parents », dans S. K. Thurman (dir.), Children of Handicapped Parents: Research and Clinical Perspectives, Orlando, Academic Press, p. 11-34.

Glaun, D.E., et P.F. Brown (1999). « Motherhood, intellectual disability and child protection: Characteristics of a court sample », Journal of Intellectual and Developmental Disability, vol. 24, $n^{\circ} 1$, p. 95-105

Goodman, S.H., et E.C. Tully (2006). « Depression in women who are mothers: An integrative model of risk for the development of psychopathology in their sons and daughters », dans C.L.M. Keyes et S.H. Goodman (dir.), Women and Depression: A Handbook for the Social, Behavioral, and Biomedical Sciences, New York, Cambridge University Press, p. 241-282.

Guay, F., L.S. Éthier, E. Palacio-Quintin et M. Boutet, M. (1997). « L'impact de la déficience intellectuelle sur la problématique de la négligence parentale », Revue européenne du handicap mental, vol. 4, $\mathrm{n}^{\circ} 15, \mathrm{p} .3-15$.

Guillemette, F., et D. Boisvert (2003). «L'entrevue de recherche qualitative avec des adultes présentant une déficience intellectuelle », Recherches qualitatives, vol. 23, p. 15-26.

Guinea, S.M. (2001). «Parents with a learning disability and their views on support received: A preliminary study », Journal of Learning Disabilities, vol. $5, \mathrm{n}^{\circ} 1$, p. 43-56.

Hayman, R. (1990). «Presumptions of justice: Law, politics and the mentally retarded parent », Harvard Law Review, vol. 103, n 6, p. 1201-1271.

Hertz, R.A. (1979). «Retarded parents in neglect proceedings: The erroneous assumption of parental inadequacy », Standford Law Review, vol. 31, n 4, p. 785-805.

Hewitt, O. (2007). «What is the effect on a child of having parent with learning disability? », Tizard Learning Disability Review, vol. 12, $\mathrm{n}^{\circ} 2$, p. 33-44.

Hunt, J., A. Macleod et C. Thomas (1999). The Last Resort: Child Protection, the Courts and the 1989 Children Act, Londres, The Stationery Office. 
Hur, J. (1997). « Review of research on parent training for parents with intellectual disability: Methodological issues », International Journal of Disability, Development and Education, vol. 44, no 2, p. 147-162.

Hyppolite, S.-R. (2012). Comprendre et agir autrement pour viser l'équité en santé dans la région de la Capitale-Nationale: Rapport du directeur régional de santé publique sur les inégalités sociales de santé, Québec, Agence de la santé et des services sociaux de la Capitale-Nationale.

Jöreskog, K., et M. Starke (2013). « Professionals' perceptions of and approach to parents with intellectual disability: A question of knowledge? », International Journal of Social Sciences Studies, vol. 1, $\mathrm{n}^{\circ}$ 2, p. 20-30.

Juhel, J.-C. (2000). La déficience intellectuelle: Connaître, comprendre, intervenir (6 ${ }^{\mathrm{e}}$ éd.), Sainte-Foy, Presses de l'Université Laval.

Julien-Gauthier, F., C. Jourdan-Ionescu et J. Héroux (2009). « Favoriser la participation des personnes ayant une déficience intellectuelle lors d'une recherche », Revue francophone de la déficience intellectuelle, vol. 20, p. 178-188.

Keltner, B.R. (1992). « Caregiving by mothers with mental retardation », Family \& Community Health, vol. 15, $n^{\circ} 2$, p. 10-18.

Khemka, I., et L. Hickson (2000). «Decision-making by adults with mental retardation in simulated situations of abuse », Mental Retardation, vol. 38, n 1, p. 15-26.

Lalande, D., L.S. Éthier, C. Rivest et M. Boutet (2002). «Parentalité et incapacités intellectuelles: Une étude pilote », Revue francophone de la déficience intellectuelle, vol. 13, $\mathrm{n}^{0} 2$, p. 133-154.

Leroux, V., et R. Scelles (2007). « Ce que disent les personnes déficientes de leur parentalité », Reliance: Revue des situations de handicap, de l'éducation et des sociétés, vol. $26, \mathrm{n}^{\circ} 4, \mathrm{p}$. 79-87.

Levesque, R.J.R. (1996). «Maintaining children's relations with mentally retarded parents: Recognizing difference and the difference that it makes », Children's Legal Rights Journal, vol. 16, $\mathrm{n}^{\circ} 2$, p. 14-22.

Lightfoot, E., K. Hill et T. LaLiberte (2010). "The inclusion of disability as a condition for termination of parental rights », Child Abuse and Neglect, vol. 34, n²12, p. 927-934.

Lightfoot, E., et T. LaLiberte (2011). «Parental supports for parents with intellectual and developmental disabilities », Intellectual and Developmental Disabilities, vol. 49, n 5, p. 388391.

Llewellyn, G. (1995). «Relationship and social support: views of parents with mental retardation/intellectual disability», Mental Retardation, vol. 33, nº 6, p. 349-363.

Llewellyn, G., et D. Bridgen (1995). «Factors affecting service provision to parents with intellectual disability: An exploratory study », Australia and New Zealand Journal of Developmental Disabilities, vol. 20, nº 2, p. 97-112. 
Llewellyn, G., et D. McConnell (2002). « Mothers with learning difficulties and their support networks », Journal of Intellectual Disability Research, vol. 46, n 1, p. 17-34.

Llewellyn, G., et D. McConnell (2010). « You have to prove yourself all the time: People with learning disabilities as parents », dans G. Grant, P. Goward, M. Richardson et P. Ramcharan (dir.), Learning Disability: A Life Cycle Aapproach to Valuing People, Maidenhead (NY), Open University Press, p. 441-467.

Llewellyn, G., D. McConnell, R. Cant et M. Westbrook (1999). « Support network of mothers with an intellectual disability: An exploratory study », Journal of Intellectual and Developmental Disability, vol. 24, $\mathrm{n}^{\circ}$ 1, p. 7-26.

Llewellyn, G., D. McConnell et L. Ferronato (2003). « Prevalence and outcomes for parents with disabilities and their children in an Australian court sample», Child Abuse and Neglect, vol. $27, n^{\circ} 3$, p. 235-251.

Llewellyn, G., D. McConnell et R. Mayes (2003). «Health of mothers with intellectual limitations », Australian and New Zealand Journal of Public Health, vol. 27, nº 1, p. 17-19.

Llewellyn, G., D. McConnell, D. Russo, R. Mayes et A. Honey (2002). « Home-based programs for parents with intellectual disabilities: Lessons from practice », Journal of Applied Research in Intellectual Disabilities, vol. 15, $\mathrm{n}^{\circ} 4$, p. 341-353.

Llewellyn, G., R. Traustadóttir, D. McConnell et H.B. Sigurjónsdóttir (dir.) (2010). Parents with Intellectual Disabilities: Past, Present and Futures, West Sussex, John Wiley \& Sons.

Lynch, E.W., et S. Bakley (1989). «Serving young children whose parents are mentally retarded », Infants and Young Children, vol. 1, n³, p. 26-38.

Lyons-Ruth, K., D. Connell, H. Grunebaum et S. Botein (1990). « Infants at social risk: Maternal depression and family support services as mediators of infant development and security of attachment », Child Development, vol. 61, n 1, p. 85-98.

MacLean, K. et M. Aunos (2010). « Addressing the needs of parents with intellectual disabilities: Exploring a parenting pilot project », Journal on Developmental Disabilities, vol. 16, $\mathrm{n}^{\circ} 1, \mathrm{p}$. 18-33.

Marx, F. (2008). « De l'importance du réseau », dans M. Mercier et G. Brazier (dir.), Parentalité des personnes déficientes mentales, Namur, Presses universitaires de Namur, p. 47-49.

Mayes, R., et G. Llewellyn (2009). « What happens to parents with intellectual disability following removal of their child in child protection proceedings? », Journal of Intellectual and Developmental Disability, vol. 34, $\mathrm{n}^{\circ}$ 1, p. 92-95.

Mayes, R., G. Llewellyn et D. McConnell (2006). « Misconception: The experience of pregnancy for women with intellectual disabilities », Scandinavian Journal of Disability Research, vol. 8, $n^{\text {os }} 2-3, p$. 120-131.

Mayes, R. et H.B. Sigurjóndóttir (2010). « Becoming a mother - becoming a father », dans G. Llewellyn, R. Traustadóttir, D. McConnell et H. B. Sigurjónsdóttir (dir.), Parents with Intellectual Disabilities: Past, Present and Futures, West Sussex, John Wiley \& Sons, p. 1733. 
McBrien, J. et M. Power (2002). «Professional attitudes to supporting parents with learning disabilities », Tizard Learning Disability Review, vol. 7, n³, p. 16-22.

McConnell, D. (2008). « Parents labeled with intellectual disability: Position of the IASSID SIRG on parents and parenting with intellectual disabilities », Journal of Applied Research in Intellectual Disabilities, vol. 21, nº 4, p. 296-307.

McConnell, D., M. Feldman, M. Aunos et N. Prasad (2011). « Child maltreatment investigations involving parents with cognitive impairments in Canada », Child Maltreatment, vol. 16, $\mathrm{n}^{\circ} 1, \mathrm{p}$. 21-32.

McConnell, D., et G. Llewellyn (2000). « Disability and discrimination in statutory child protection proceedings », Disability \& Society, vol. $15, \mathrm{n}^{\circ} 6$, p. 883-895.

McConnell, D., et G. Llewellyn (2002). « Stereotypes, parents with intellectual disability and child protection », Journal of Social Welfare and Family Law, vol. 24, n³ 3, p. 297-317

McConnell, D., G. Llewellyn et L. Ferronato (2000). Parents with a Disability and the NSW Children's Court, Lidcombe, University of Sydney, Family support and services project.

McConnell, D., G. Llewellyn et L. Ferronato (2002). «Disability and decision making in Australian care proceedings », International Journal of Law, Policy and the Family, vol. 16, $\mathrm{n}^{\circ} 2$, p. $270-299$.

McConnell, D., G. Llewellyn et L. Ferronato (2006). « Context-contingent decision-making in child protection practice », International Journal of Social Welfare, vol. 15, $\mathrm{n}^{\circ}$ 3, p. 230-239.

McConnell, D., et H.B. Sigurjónsdóttir (2010). « Caught in the child protection net », dans G. Llewellyn, R. Traustadóttir, D. McConnell et H. B. Sigurjónsdóttir, Parents with Intellectual Disabilities: Past, Present and Futures, West Sussex, John Wiley \& Sons,p. 171-187.

McGraw, S., T. Scully et C. Pritchard (2010). «Predicting the unpredictable? Identifying high risk versus low-risk parents with intellectual disabilities », Child Abuse and Neglect, vol. 34, $n^{\circ} 9$, p. 699-710.

McGraw, S., T. Shaw et K. Beckley (2007). «Prevalence of psychopathology across a service population of parents with intellectual disabilities and their children », Journal of Policy and Practice in Intellectual Disabilities, vol. 4, $\mathrm{n}^{\circ} 1$, p. 11-22.

Mildon, R., J.M. Matthews et S. Gavidia-Payne (2003). Best Practice in Parenting Education: Understanding and Supporting Parents with Learning Difficulties, Melbourne, Victorian parenting centre.

Ministère de la Santé et des Services sociaux (MSSS) (2007). Un projet de vie, des racines pour la vie, Québec, MSSS.

National Council on Disability (NCIS) (2012). Rocking the Cradle: Ensuring the Rights of Parents with Disabilities and Their Children. Washington, NCIS.

O'Neill, A. M. (1985). « Normal and bright children of mentally retarded parents: The Huck Finn syndrome », Child Psychiatry \& Human Development, vol. 15, n 4, p. 255-268. 
O'Neill, A. M. (2011). « Average and bright adults with parents with mild cognitive difficulties: The Huck Finn syndrome 20 years later », Journal of Applied Research in Intellectual Disabilities, vol. 24, $\mathrm{n}^{\circ} 6$, p. 566-572.

Organisation des Nations Unies (ONU) (2006). Convention relative aux droits des personnes handicapées. Genève, ONU. Disponible en ligne <http://www.un.org/esa/socdev/enable/documents/tccconvf.pdf>.

Picard, S., C. Loyer et C. Mercier (2011). "Itinérance et limitations intellectuelles: Problématiques associées et intervention », Revue francophone de la déficience intellectuelle, vol. 22, p. 14-19.

Pixa-Kettner, U. (1999). « Follow-up study on parenting with intellectual disability in Germany », Journal of Intellectual and Developmental Disability, vol. 24, nº 1, p. 75-93.

Ray, N., H. Rubenstein et N. Russo (1994). « Understanding the parents who are mentally retarded: Guidelines for family preservation programs », Child Welfare, vol. 73, nº, p. 725743.

Seagull, E.A.W. et S.L. Scheurer (1986). « Neglected and abused children of mentally retarded parents », Child Abuse and Neglect, vol. 10, n 4, p. 493-500.

Sobsey, D. (2000). « Faces of violence against women with developmental disabilities », Impact, vol. $13, n^{\circ} 3$, p. $2-27$.

Sobsey, D., et T. Doe (1991). «Patterns of sexual abuse and assault », Sexuality and Disability, vol. $9, n^{\circ} 3$, p. 243-259.

St-Amand, K., M. Aunos et G. Goupil (2010a). «Perceptions de dix mères présentant une déficience intellectuelle sur le soutien reçu et souhaité », Revue francophone de la déficience intellectuelle, vol. 21, p. 110-124.

St-Amand, K., G. Goupil et M. Aunos (2010b). « Mères présentant une déficience intellectuelle : Perceptions de leur qualité de vie », Journal on Developmental Disabilities, vol. 16, $\mathrm{n}^{\circ} 3, \mathrm{p}$. 18-27.

Starke, M. (2010). «Encounters with professionals: Views and experiences of mothers with intellectual disability », Journal of Intellectual Disabilities, vol. 14, nº 1, p. 9-19.

Strike, R., et D. McConnell (2002). « Look at me, listen to me, I have something important to say », Sexuality and Disability, vol. $20, \mathrm{n}^{0} 1$, p. 53-63.

Swain, P.A., et N. Cameron (2003). « "Good enough parenting": Parental disability and child protection », Disability \& Society, vol. $18, \mathrm{n}^{\circ} 2$, p. 165-177.

Tarleton, B., et L. Ward (2007). " "Parenting with support": The views and experiences of parents with intellectual disabilities », Journal of Policy and Practice in Intellectual Disabilities, vol. $4, n^{0} 3$, p. 194-202.

Tarleton, B., L. Ward et J. Howarth (2006). Finding the Support? A Review of Issues and Positive Practice in Supporting Parents with Learning Difficulties and Their Children, Londres, Baring Foundation. 
Taylor, J.L., H.D. Hurd, M.M. Seltzer, J.S. Greenberg et F.J. Floyd (2010). « Parenting with mild intellectual deficits: Parental expectations and the educational attainment of their children », American Journal on Intellectual and Developmental Disabilities, vol. 115, $n^{\circ} 4$, p. 340-354.

Tharinger, D., C.B. Horton et S. Millea (1990). « Sexual abuse and exploitation of children and adults with mental retardation and other handicaps », Child Abuse and Neglect, vol. 14, p. 301-312.

Tymchuk, A.J. (1992). «Predicting adequacy of parenting by people with mental retardation », Child Abuse and Neglect, vol. 16, $\mathrm{n}^{\circ}$ 2, p. 165-178.

Tymchuk, A.J. (1994). « Depression symptomatology in mothers with mild intellectual disability: An exploratory study », Australia and New Zealand Journal of Developmental Disabilities, vol. $19, \mathrm{n}^{\circ} 2$, p. 111-119.

Tymchuk, A.J. (1999). « Moving towards integration of services for parents with intellectual disabilities », Journal of Intellectual and Developmental Disability, vol. 24, no 1, p. 59-74.

Tymchuk, A.J. et M. Feldman (1991). «Parents with mental retardation and their children: Review of research relevant to professional practice », Canadian Psychology, vol. 32, $n^{\circ} 3, p$. 486-496.

Tymchuk, A.J., D. Hamada, L. Andron et S. Anderson (1990a). «Emergency training with mothers who are mentally retarded », Child and Family Behavior Therapy, vol. 12, $n^{\circ} 3$, p. 3147.

Tymchuk, A.J., A. Yokota et B. Rahbar (1990b). «Decision-making abilities of mothers with mental retardation », Research in Developmental Disabilities, vol. 11, n 1, p. 97-109.

Vroman, A.-M. (2008). «L'identité du parent », dans M. Mercier et G. Brazier, Parentalité des personnes déficientes mentales, Namur, Presses universitaires de Namur, p. 27-32.

Wade, C., G. Llewellyn et J.M. Matthews (2008). «Review of parent training interventions for parents with intellectual disability», Journal of applied research in intellectual disabilities, vol. 21, p. 351-366.

Wade, C., G. Llewellyn et J.M. Matthews (2011). « Modeling contextual influences on parents with intellectual disability and their children », American Journal on Intellectual and Developmental Disabilities, vol. 116, $\mathrm{n}^{\circ}$ 6, p. 419-437.

Wade, C. M., R.L. Mildon et J.M. Matthews (2007). «Service delivery to parents with an intellectual disability: Family-centred or professionally centred?», Journal of applied research in intellectual disabilities, vol. 20, p. 87-98.

Webster-Stratton, C. (1997). « From parent training to community building », Families in Society, vol. 78, p. 156-171.

Webster-Stratton, C. (1998). «Parent training with low-income families: Promoting parental engagement through a collaborative approach », dans J.R. Lutzker (dir.), Handbook of Child Abuse Research and Treatment, New York, Kluwer Academic/Plenum Press, p. 183-210. 
Whitman, B.Y., et P.J. Accardo (dir.) (1990). When a Parent Is Mentally Retarded, Baltimore, Paul H. Brookes publishing co.

Wilson, C., et N. Brewer (1992). « The incidence of criminal victimization of individuals with an intellectual disability », Australian Psychologist, vol. 27, n² 2, p. 114-117. 